\title{
SPATIALITY OF ISOMORPHISMS BETWEEN CERTAIN REFLEXIVE ALGEBRAS
}

\author{
M. S. LAMBROU AND W. E. LONGSTAFF
}

(Communicated by Palle E. T. Jorgensen)

\begin{abstract}
Two subspaces $M$ and $N$ of a Hilbert space $H$ are in generalized generic position if $M \cap N=M^{\perp} \cap N^{\perp}=(0)$ and $\operatorname{dim}\left(M^{\perp} \cap N\right)$ $=\operatorname{dim}\left(M \cap N^{\perp}\right)$. If $H$ is separable and both the pairs $\left\{M_{1}, N_{1}\right\}$ and $\left\{M_{2}, N_{2}\right\}$ are in generalized generic position, then every algebraic isomorphism $\varphi: \operatorname{Alg}\left\{M_{1}, N_{1}\right\} \rightarrow \operatorname{Alg}\left\{M_{2}, N_{2}\right\}$ is spatially induced, that is, there exists an invertible operator $T_{0} \in \mathscr{B}(H)$ such that $\varphi(B)=T_{0} B T_{0}^{-1}$, for every $B \in \operatorname{Alg}\left\{M_{1}, N_{1}\right\}$. The proof of this uses the following result: If $H$ is separable, $\mathscr{M} \subseteq H$ is a proper operator range in $H$, and the operator $T \in \mathscr{B}(H)$ has the property that, for every $W \in \mathscr{B}(H)$ leaving $\mathscr{M}$ invariant, the range of $W T-T W$ is included in $\mathscr{K}$, then the range of $T-\lambda$ is included in $\mathscr{M}$, for some unique scalar $\lambda$.
\end{abstract}

\section{INTRODUCTION AND PRELIMINARIES}

In the terminology of Halmos [3] two subspaces $M$ and $N$ of a Hilbert space $H$ are said to be in generic position if $M \cap N=M^{\perp} \cap N=M \cap N^{\perp}=M^{\perp} \cap N^{\perp}=$ (0). Following Papadakis [7] we will say that $M$ and $N$ are in generalized generic position if $M \cap N=M^{\perp} \cap N^{\perp}=(0)$ and $\operatorname{dim}\left(M^{\perp} \cap N\right)=\operatorname{dim}\left(M \cap N^{\perp}\right)$. Papadakis [7] has recently shown that, if $\left\{M_{1}, N_{1}\right\}$ and $\left\{M_{2}, N_{2}\right\}$ are pairs of subspaces with $M_{i}$ and $N_{i}$ in generalized generic position $(i=1,2)$, then the corresponding operator algebras $\operatorname{Alg}\left\{M_{1}, N_{1}\right\}$ and $\operatorname{Alg}\left\{M_{2}, N_{2}\right\}$ are spatially isomorphic whenever they are algebraically isomorphic. The question then naturally arises as to whether or not every algebraic isomorphism $\varphi: \operatorname{Alg}\left\{M_{1}, N_{1}\right\} \rightarrow \operatorname{Alg}\left\{M_{2}, N_{2}\right\}$ must itself be spatially induced. We prove below that the answer is affirmative, at least on separable space. The proof uses a result (Theorem 1), perhaps of some independent interest, concerning the algebra of operators leaving a given fixed operator range invariant. The proof of the latter result uses some facts about derivations.

Throughout what follows, all Hilbert spaces will be over the complex field of scalars, and inner-products will be denoted by $(\cdot \mid \cdot)$. Also, "operator" will mean "bounded linear operator", and "subspace" will mean "closed linear subspace". For Hilbert spaces $H_{1}$ and $H_{2}, \mathscr{B}\left(H_{1}, H_{2}\right)$ will denote the set of all

Received by the editors March 5, 1993.

1991 Mathematics Subject Classification. Primary 47A15; Secondary 47B05, 47B47.

This research was supported by the Australian Research Council. 
operators mapping $H_{1}$ into $H_{2}$ and $\mathscr{B}(H)$ will be used in place of $\mathscr{B}(H, H)$. The set of compact operators on $H$ will be denoted by $\mathscr{K}(H)$. If $\mathscr{A}_{1}$ and $\mathscr{A}_{2}$ are subalgebras of $\mathscr{B}(H)$, an algebraic isomorphism of $\mathscr{A}_{1}$ onto $\mathscr{A}_{2}$ is a linear, multiplicative bijection $\varphi: \mathscr{A}_{1} \rightarrow \mathscr{A}_{2}$. Such a mapping is said to be spatially induced if there exists an invertible operator $T_{0} \in \mathscr{B}(H)$ such that $\varphi(B)=T_{0} B T_{0}^{-1}$, for every $B \in \mathscr{A}_{1}$. For any family $\mathfrak{L}$ of subspaces of $H$ we let $\mathrm{Alg} \mathfrak{L}$ denote the algebra of operators leaving every member of $\mathfrak{L}$ invariant, that is,

$$
\operatorname{Alg} \mathfrak{L}=\{T \in \mathscr{B}(H): T(L) \subseteq L, \text { for every } L \in \mathfrak{L}\} .
$$

For any operator $A \in \mathscr{B}(H), \mathscr{R}(A)$ will denote its range and $G(A)$ its graph. Thus $G(A)$ is the subset of $H \oplus H$ given by $G(A)=\{(x, A x): x \in H\}$. A linear manifold $\mathscr{H} \subseteq H$ is an operator range in $H$ if $\mathscr{M}=\mathscr{R}(A)$ for some $A \in \mathscr{B}(H)$. Most of the basic results about operator ranges that we use are taken from [2]. In particular, we use in several ways the range inclusion theorem of Douglas (see [2, Theorem 2.1]): Let $A, B \in \mathscr{B}(H)$. Then $\mathscr{R}(A) \subseteq \mathscr{R}(B)$ if and only if $A=B C$, for some $C \in \mathscr{B}(H)$. In particular, if $A \in \mathscr{B}(H)$, then $W \in \mathscr{B}(H)$ leaves $\mathscr{R}(A)$ invariant if and only if $W A=A X$, for some $X \in \mathscr{B}(H)$. Following [6] we let $\mathscr{A}(A)$ denote the algebra of all operators leaving $\mathscr{R}(A)$ invariant. Thus $\mathscr{A}(A)=\{W \in \mathscr{B}(H): W A=A X$, for some $X \in \mathscr{B}(H)\}$. Notice that, if $A$ is injective and $W \in \mathscr{A}(A)$, then $A^{-1} W A$ is bounded; if, additionally, $A$ is selfadjoint, then $\left\{\left(A^{-1} W A\right)^{*}: W \in \mathscr{A}(A)\right\}=$ $\mathscr{A}(A)$.

Frequently, for the sake of simplicity, and when no confusion is likely to arise, we will identify an operator on $H$ with its matrix representation relative to a given orthogonal decomposition of $H$.

\section{Main Results}

The following result, perhaps of some independent interest, will be used later. The authors thank H. Radjavi for helpful discussions concerning its proof.

Theorem 1. Let $H$ be a separable Hilbert space, let $\mathscr{M} \subseteq H$ be a proper operator range in $H$, and let $T \in \mathscr{B}(H)$. If $\mathscr{R}(W T-T W) \subseteq \mathscr{M}$, for every operator $W \in \mathscr{B}(H)$ leaving $\mathscr{M}$ invariant, then $\mathscr{R}(T-\lambda) \subseteq \mathscr{M}$, for some unique scalar $\lambda$.

Proof. The uniqueness of $\lambda$ follows immediately from the fact that $\mathscr{M}$ is proper. We turn to the question of its existence.

First, suppose that $\mathscr{M}$ is not dense (this, of course, includes the case where $\mathscr{M}$ is closed). Then $H$ decomposes as $H=\overline{\mathscr{M}} \oplus \overline{\mathscr{M}}^{\perp}$ (where $\overline{\mathscr{M}}$ denotes the norm closure of $\mathscr{M}$ ), with $\overline{\mathscr{M}}^{\perp} \neq(0)$. Relative to this decomposition, let $T=\left(\begin{array}{ll}T_{1} & T_{2} \\ T_{3} & T_{4}\end{array}\right)$ and let $T$ satisfy the condition in the statement of the theorem, namely,

(1) $\mathscr{R}(W T-T W) \subseteq \mathscr{M}, \quad$ for every $W \in \mathscr{B}(H)$ leaving $\mathscr{M}$ invariant .

If $U \in \mathscr{B}\left(\overline{\mathscr{M}}^{\perp}\right)$ and $V \in \mathscr{B}\left(\overline{\mathscr{M}}^{\perp}, \overline{\mathscr{M}}\right)$, the operators $\left(\begin{array}{ll}1 & 0 \\ 0 & 0\end{array}\right),\left(\begin{array}{ll}0 & 0 \\ 0 & U\end{array}\right),\left(\begin{array}{ll}0 & V \\ 0 & 0\end{array}\right)$ leave $\mathscr{M}$ invariant. Taking $W=\left(\begin{array}{ll}1 & 0 \\ 0 & 0\end{array}\right)$ in (1) gives $\mathscr{R}\left(\left(\begin{array}{cc}0 & T_{2} \\ -T_{3} & 0\end{array}\right)\right) \subseteq \mathscr{M}$, so $T_{3}=0$ and $\mathscr{R}\left(T_{2}\right) \subseteq \mathscr{M}$. Taking $W=\left(\begin{array}{ll}0 & 0 \\ 0 & U\end{array}\right)$ in $(1)$ gives $\mathscr{R}\left(\left(\begin{array}{ll}0 & -T_{2} U \\ 0 & U T_{4}-T_{4} U\end{array}\right)\right) \subseteq$ $\mathscr{M}$ and it follows that $T_{4}=\lambda$, for some scalar $\lambda$. Taking $W=\left(\begin{array}{ll}0 & V \\ 0 & 0\end{array}\right)$ in 
(1) gives $\mathscr{R}\left(\left(\begin{array}{cc}0 & \left(\lambda-T_{1}\right) V \\ 0 & 0\end{array}\right)\right) \subseteq \mathscr{M}$, so $\mathscr{R}\left(T_{1}-\lambda\right) \subseteq \mathscr{M}$. Hence $\mathscr{R}(T-\lambda)=$ $\mathscr{R}\left(\left(\begin{array}{cc}T_{1}-\lambda & T_{2} \\ 0 & 0\end{array}\right) \subseteq \mathscr{M}\right.$.

Next, suppose that $\mathscr{M}$ is dense (then, of course, $H$ is infinite-dimensional). Now $\mathscr{M}=\mathscr{R}(A)$ for some positive, injective, noninvertible operator $A \in$ $\mathscr{B}(H)$ [2] and we may suppose that $\|A\|=1$. The range inclusion theorem of Douglas reduces what we have to prove to showing that, if $T \in \mathscr{B}(H)$ satisfies:

$$
\begin{aligned}
& \text { For every } W \in \mathscr{A}(A), \\
& \text { there exists } C \in \mathscr{B}(H) \text { such that } W T-T W=A C,
\end{aligned}
$$

then there exists a scalar $\lambda$ and an operator $R \in \mathscr{B}(H)$ such that $T-\lambda=A R$. Here $\mathscr{A}(A)=\{W \in \mathscr{B}(H): W A=A X$, for some $X \in \mathscr{B}(H)\}$. Let $T$ satisfy (2).

Suppose that $A$ is compact. In (2), since $A$ is injective, the operator $C$ is uniquely determined by $W$. This defines a mapping $\delta: \mathscr{A}(A) \rightarrow \mathscr{B}(H)$ given by $\delta(W)=C$. This mapping is obviously linear. Let $X, Y \in A^{\prime}$, the commutant of $A$. Then $X T-T X=A \delta(X)$ and $Y T-T Y=A \delta(Y)$. Multiplying the first of these equations on the right by $Y$ and the second on the left by $X$ and then adding, we obtain $(X Y) T-T(X Y)=A(\delta(X) Y+X \delta(Y))$. Hence $\delta: A^{\prime} \rightarrow \mathscr{B}(H)$ is a derivation. For such a derivation (see [1, Lemma 8.4, p. 90 and Theorem 10.8, p. 114]) there exists an operator $R_{1} \in \mathscr{B}(H)$ such that $\delta(X)=X R_{1}-R_{1} X$, for every $X \in A^{\prime}$. Since $X T-T X=A\left(X R_{1}-R_{1} X\right)=$ $X\left(A R_{1}\right)-\left(A R_{1}\right) X$, for every $X \in A^{\prime}$, the operator $T_{1}=T-A R_{1}$ belongs to $A^{\prime \prime}$. Moreover, (2) holds with $T$ replaced by $T_{1}$. Since $A$ is compact, the range of the mapping $\delta_{T_{1}}: \mathscr{A}(A) \rightarrow \mathscr{B}(H)$ defined by $\delta_{T_{1}}(W)=W T_{1}-T_{1} W$ is included in $\mathscr{K}(H)$, the set of compact operators on $H$. Now $\mathscr{A}(A)$ contains a nest algebra $\mathscr{T}[6]$ and $\delta_{T_{1}}$ maps $\mathscr{T}$ into $\mathscr{K}(H)$. It follows (see [1, Theorem 19.12, p. 263]) that there exists an operator $K \in \mathscr{K}(H)$ such that $\delta_{T_{1}}(W)=$ $W K-K W$, for every $W \in \mathscr{T}$. Hence $T_{1}-K \in \mathscr{T}^{\prime}$ and since $\mathscr{T}^{\prime}=\mathbb{C I}$ (see [1, Corollary 19.5 , p. 260]), $T_{1}=\lambda+K$, for some $\lambda \in \mathbb{C}$. We show that $K=A R_{2}$, for some $R_{2} \in \mathscr{B}(H)$. Then $T-\lambda=A R$ with $R=R_{1}+R_{2}$.

Relative to some orthonormal basis $\left(e_{n}\right)_{1}^{\infty}$ of $H, A=\operatorname{diag}\left(a_{1}, a_{2}, a_{3}, \ldots\right)$ where $a_{i}>0$, for every $i \geq 1$ and where $a_{i} \rightarrow 0$. Since $K \in A^{\prime \prime}, K=$ $\operatorname{diag}\left(b_{1}, b_{2}, b_{3}, \ldots\right)$ relative to this basis, and $b_{i} \rightarrow 0$ by compactness. Let $\left(n_{i}\right)_{1}^{\infty}$ be an increasing sequence of positive integers such that both of the sequences $\left(a_{n_{i}} / a_{i}\right)_{1}^{\infty}$ and $\left(b_{n_{i}} / a_{i}\right)_{1}^{\infty}$ are bounded. Let $W=\left(w_{i j}\right)$ relative to $\left(e_{n}\right)_{1}^{\infty}$, where

$$
w_{i j}= \begin{cases}1, & \text { if } j=n_{i}, \\ 0, & \text { if } j \neq n_{i} .\end{cases}
$$

Since $\left(a_{n_{i}} / a_{i}\right)_{1}^{\infty}$ is bounded, $W \in \mathscr{A}(A)$ (the $(i, j)$ th entry of $A^{-1} W A$ is $a_{n_{i}} / a_{i}$ if $j=n_{i}$, and zero otherwise). Since $W K-K W=A C$, for some $C \in \mathscr{B}(H),\left(\left(b_{i}-b_{n_{i}}\right) / a_{i}\right)_{1}^{\infty}$ is bounded (the $(i, j)$ th entry of $C$ is $\left(b_{n_{i}}-b_{i}\right) / a_{i}$ if $j=n_{i}$, and zero otherwise). Since $b_{i} / a_{i}=\left(b_{i}-b_{n_{i}}\right) / a_{i}+b_{n_{i}} / a_{i}$, it follows that $\left(b_{i} / a_{i}\right)_{1}^{\infty}$ is bounded. Thus $K=A R_{2}$ where $R_{2}=\operatorname{diag}\left(b_{1} / a_{1}, b_{2} / a_{2}, \ldots\right)$.

Next, suppose that $A$ is not compact but that there exist Hilbert spaces $H_{1}$ and $H_{2}$ such that $A$ is unitarily equivalent to an operator of the form $\left(\begin{array}{ll}U & 0 \\ 0 & K\end{array}\right)$ on $H_{1} \oplus H_{2}$ with $U \in \mathscr{B}\left(H_{1}\right)$ invertible and $K \in \mathscr{B}\left(H_{2}\right)$ compact (necessarily, both $H_{1}$ and $H_{2}$ are infinite dimensional). In fact, we can suppose 
that $A=\left(\begin{array}{cc}U & 0 \\ 0 & K\end{array}\right)$. Let $T=\left(\begin{array}{ll}T_{1} & T_{2} \\ T_{3} & T_{4}\end{array}\right) \in \mathscr{B}\left(H_{1} \oplus H_{2}\right)$ satisfy:

$$
\begin{gathered}
\text { For every } W=\left(\begin{array}{ll}
W_{1} & W_{2} \\
W_{3} & W_{4}
\end{array}\right) \in \mathscr{A}(A), \text { there exists } C \in \mathscr{B}\left(H_{1} \oplus H_{2}\right) \\
\text { such that } W T-T W=A C .
\end{gathered}
$$

Then, taking $W=\left(\begin{array}{ll}1 & 0 \\ 0 & 0\end{array}\right)$ in (3) gives that $T_{3}=K C_{3}$, for some $C_{3} \in \mathscr{B}\left(H_{1}, H_{2}\right)$. Taking $W=\left(\begin{array}{ll}0 & 0 \\ 0 & V\end{array}\right)$ in (3), where $V \in \mathscr{A}(K)$, gives that $V T_{4}-T_{4} V=K C_{4}$, for some $C_{4} \in \mathscr{B}\left(H_{2}\right)$. By what has already been proved, it follows that $T_{4}-\lambda=K R_{4}$, for some scalar $\lambda$ and some operator $R_{4} \in \mathscr{B}\left(H_{2}\right)$. Hence

$$
\left(\begin{array}{ll}
T_{1} & T_{2} \\
T_{3} & T_{4}
\end{array}\right)-\left(\begin{array}{ll}
\lambda & 0 \\
0 & \lambda
\end{array}\right)=\left(\begin{array}{cc}
U & 0 \\
0 & K
\end{array}\right)\left(\begin{array}{cc}
U^{-1}\left(T_{1}-\lambda\right) & U^{-1} T_{2} \\
C_{3} & R_{4}
\end{array}\right),
$$

which is the desired result.

Finally, suppose that $A$ is not compact and not unitarily equivalent to an operator of the form $\left(\begin{array}{ll}U & 0 \\ 0 & K\end{array}\right)$ with $U$ invertible and $K$ compact. Let $E$ be the spectral measure of $A$ and let $a \in(0,1)$. There exists an increasing sequence $\left(m_{i}\right)_{1}^{\infty}$ of natural numbers, with $m_{1}=0$, such that each of the spaces $H_{i}=E\left(\left(a^{m_{i+1}}, a^{m_{i}}\right]\right)$, for $i \geq 1$, is infinite dimensional. Let $A_{i}=\left.A\right|_{H_{i}}$, for $i \geq 1$. Then, for every $i \geq 1, A_{i}$ is a positive invertible operator and $a^{m_{i+1}} \leq A_{i} \leq a^{m_{i}}$, so $a^{m_{i+1}} \leq\left\|A_{i}\right\| \leq a^{m_{i}}$. Relative to the decomposition $H=\bigoplus_{i=1}^{\infty} H_{i}, A=\operatorname{diag}\left(A_{1}, A_{2}, A_{3}, \ldots\right)$. We can suppose that, for every $i \geq 1, H_{i}=H_{0}$ where $H_{0}$ is a separable, infinite-dimensional Hilbert space. Then $H=H_{0}^{(\infty)}$. Let $T \in \mathscr{B}(H)$ satisfy (2). Let $S$ be the operator on $H_{0}^{(\infty)}$ given by

$$
S=\left(\begin{array}{cccc}
0 & 0 & 0 & \ldots \\
I & 0 & 0 & \ldots \\
0 & I & 0 & \ldots \\
\cdot & \cdot & \cdot & \ldots \\
\cdot & \cdot & . & \ldots \\
. & . & . & \ldots
\end{array}\right)
$$

Then $S^{*} A=A V$ where

$$
V=\left(\begin{array}{ccccc}
0 & A_{1}^{-1} A_{2} & 0 & 0 & \cdots \\
0 & 0 & A_{2}^{-1} A_{3} & 0 & \ldots \\
0 & 0 & 0 & A_{3}^{-1} A_{4} & \ldots \\
\cdot & \cdot & \cdot & \cdot & \cdots \\
\cdot & \cdot & \cdot & \cdot & \ldots \\
\cdot & \cdot & . & . & \ldots
\end{array}\right)
$$

is a bounded operator since $\left\|A_{i}^{-1} A_{i+1}\right\| \leq\left\|A_{i}^{-1}\right\|\left\|A_{i+1}\right\| \leq 1$, for every $i \geq 1$. Thus $S^{*} \in \mathscr{A}(A)$ so $S^{*} T-T S^{*}=A C$, for some $C \in \mathscr{B}(H)$. Multiplying this last equation on the right by $S$, and noting that $S^{*} S=I$, gives $S^{*} T S-T=$ $A C S$. By induction, for every $k \geq 1$, we have $S^{* k} T S^{k}-S^{* k-1} T S^{k-1}=$ $A V^{k-1} C S^{k}$. Adding the first $n$ of these equations yields

$$
S^{* n} T S^{n}-T=A\left(\sum_{k=1}^{n} V^{k-1} C S^{k}\right) \text {. }
$$


Now, for every $k \geq 1$,

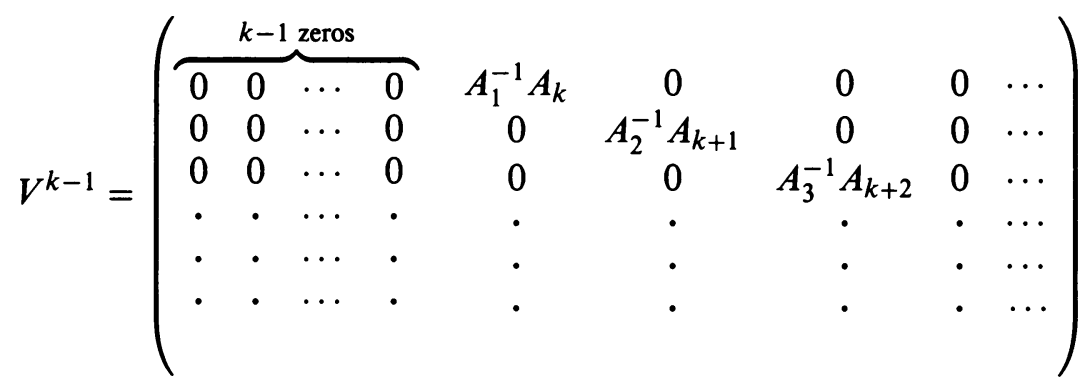

and, for every $i \geq 1,\left\|A_{i}^{-1} A_{k-1+i}\right\| \leq\left\|A_{i}^{-1}\right\|\left\|A_{k-1+i}\right\| \leq a^{m_{k-1+i}} / a^{m_{i+1}}=$ $a^{m_{k-1+i}-m_{i+1}} \leq a^{k-1+i-i-1}=a^{k-2}$, so $\left\|V^{k-1}\right\| \leq a^{k-2}$ and $\left\|V^{k-1} C S^{k}\right\| \leq$ $a^{k-2}\|C\|$ (note that $\|S\|=1$ ). It follows that the series $\sum_{k=1}^{\infty} V^{k-1} C S^{k}$ converges in operator norm. Let $R=\sum_{k=1}^{\infty} V^{k-1} C S^{k}$. Then $S^{* n} T S^{n} \rightarrow T+A R$ in norm, so $S^{* n+1} T S^{n+1} \rightarrow T+A R$. Hence, the operator $D=T+A R$ satisfies $S^{*} D S=D$. The latter equation means that $D$ has the form

$$
D=\left(\begin{array}{cccc}
D_{0} & D_{1} & D_{2} & \ldots \\
D_{-1} & D_{0} & D_{1} & \ldots \\
D_{-2} & D_{-1} & D_{0} & \ldots \\
\cdot & \cdot & \cdot & \ldots \\
\cdot & \cdot & \cdot & \ldots \\
\cdot & \cdot & \cdot & \ldots
\end{array}\right),
$$

that is, $D$ has constant (operator-valued) diagonals. We next show that $D_{p}=0$, for every $|p| \geq 1$. Let $b$ satisfy $a<b<1$. Let $W_{0}$ be the operator on $H=$ $H_{0}^{(\infty)}$ given by $W_{0}=\operatorname{diag}\left(b^{m_{1}}, b^{m_{2}}, b^{m_{3}}, \ldots\right)$. Then $W_{0} \in \mathscr{A}(A)$, and since $T$ satisfies (2), there exists an operator $C_{0} \in \mathscr{B}(H)$ such that $W_{0} D-D W_{0}=A C_{0}$. The latter gives that

$$
\left|b^{m_{i}}-b^{m_{j}}\right|\left\|A_{i}^{-1} D_{j-i}\right\| \leq\left\|C_{0}\right\|, \quad \text { for every } i, j \geq 1 .
$$

Let $p \in \mathbb{Z}^{+}$and suppose that $D_{p} \neq 0$. Then $D_{p} x \neq 0$, for some vector $x \in H_{0}$. Now, for every $i \geq 1,1 / a^{m_{i}} \leq A_{i}^{-1} \leq 1 / a^{m_{i+1}}$, so

$\left|\left(\left(b^{m_{i}}-b^{m_{i+p}}\right) A_{i}^{-1}\left(D_{p} x\right) \mid D_{p} x\right)\right| \geq\left(b^{m_{i}}-b^{m_{i+p}}\right) \frac{\left\|D_{p} x\right\|^{2}}{a^{m_{i}}} \geq\left(\frac{b}{a}\right)^{m_{i}}\left(1-b^{p}\right)\left\|D_{p} x\right\|^{2}$

and hence $\left|\left(\left(b^{m_{i}}-b^{m_{i+p}}\right) A_{i}^{-1}\left(D_{p} x\right) \mid D_{p} x\right)\right| \rightarrow \infty$ as $i \rightarrow \infty$. But this contradicts the fact that, for every $i \geq 1$,

$$
\left|\left(\left(b^{m_{i}}-b^{m_{i+p}}\right) A_{i}^{-1}\left(D_{p} x\right) \mid D_{p} x\right)\right| \leq\left\|C_{0}\right\|\|x\|\left\|D_{p} x\right\| .
$$

Hence, $D_{p}=0$, for every $p \in \mathbb{Z}^{+}$. A similar argument shows that $D_{-p}=0$, for every $p \in \mathbb{Z}^{+}$. Thus $D=\operatorname{diag}\left(D_{0}, D_{0}, D_{0}, \ldots\right)$.

Finally, we show that $D_{0}=\lambda$, for some scalar $\lambda$. Let $B \in \mathscr{B}\left(H_{0}\right)$ and put

$$
W_{1}=\left(\begin{array}{ccccc}
0 & B & 0 & 0 & \ldots \\
0 & 0 & B & 0 & \ldots \\
0 & 0 & 0 & B & \ldots \\
. & . & . & . & \ldots \\
. & . & . & . & \ldots \\
. & . & . & . & \ldots
\end{array}\right) .
$$


Then, $W_{1} \in \mathscr{A}(A)$ since $\left\|A_{i}^{-1} B A_{i+1}\right\| \leq\|B\|$, for every $i \geq 1$. Since $W_{1} D-$ $D W_{1}=A C_{1}$, for some operator $C_{1} \in \mathscr{B}(H)$, it follows that the sequence $\left(\left\|A_{i}^{-1}\left(B D_{0}-D_{0} B\right)\right\|\right)_{1}^{\infty}$ is bounded. For every vector $y \in H_{0}$ and every $i \geq 1$ we have

$$
\left(A_{i}^{-1}\left(B D_{0}-D_{0} B\right) y \mid\left(B D_{0}-D_{0} B\right) y\right) \geq\left\|\left(B D_{0}-D_{0} B\right) y\right\|^{2} / a^{m_{i}}
$$

and

$$
\left(A_{i}^{-1}\left(B D_{0}-D_{0} B\right) y \mid\left(B D_{0}-D_{0} B\right) y\right) \leq\left\|A_{i}^{-1}\left(B D_{0}-D_{0} B\right)\right\|\|y\|\left\|\left(B D_{0}-D_{0} B\right) y\right\|,
$$

so the sequence $\left(\left\|\left(B D_{0}-D_{0} B\right) y\right\|^{2} / a^{m_{i}}\right)_{1}^{\infty}$ is also bounded. Hence $B D_{0} y=$ $D_{0} B y$ and it follows that $D_{0}=\lambda$, for some scalar $\lambda$. Thus $D=\lambda$ and $T-\lambda=A(-R)$. This completes the proof of the theorem.

Let $\left\{M_{1}, N_{1}\right\}$ and $\left\{M_{2}, N_{2}\right\}$ be pairs of subspaces of a Hilbert space with $M_{i}$ and $N_{i}$ in generalized generic position $(i=1,2)$. Let $\varphi: \operatorname{Alg}\left\{M_{1}, N_{1}\right\} \rightarrow$ $\operatorname{Alg}\left\{M_{2}, N_{2}\right\}$ be an algebraic isomorphism. By [7, Theorem 2.5] there exists a spatially induced isomorphism $\psi: \operatorname{Alg}\left\{M_{1}, N_{1}\right\} \rightarrow \operatorname{Alg}\left\{M_{2}, N_{2}\right\}$. Now $\psi^{-1} \circ \varphi$ is an algebraic isomorphism of $\operatorname{Alg}\left\{M_{1}, N_{1}\right\}$ onto itself and if it were spatially induced so would $\varphi$ be, since $\varphi=\psi \circ\left(\psi^{-1} \circ \varphi\right)$ and the composite of two spatially induced isomorphisms is itself spatially induced. Since, as remarked in [7], the pair $\left\{M_{1}, N_{1}\right\}$ is unitarily equivalent to $\{G(-A), G(A)\}$ where $A$ is a positive injective contraction on some Hilbert space, it will follow that $\psi^{-1} \circ \varphi$ (as above) is spatially induced if every automorphism of $\operatorname{Alg}\{G(-A), G(A)\}$ is. The latter is proved below (Theorem 2) in the case where the underlying space is separable. It is convenient to first use the preceding theorem to prove a lemma.

Lemma. Let $H$ be a separable Hilbert space, let $A \in \mathscr{B}(H)$ be a positive injective contraction and let $P, Q \in \mathscr{B}(H)$ be invertible operators. Let $T$ be the linear transformation defined on $G(-A)+G(A)$ by

$$
T\left(\begin{array}{c}
x+y \\
A(y-x)
\end{array}\right)=\left(\begin{array}{c}
P x+Q y \\
A(Q y-P x)
\end{array}\right) .
$$

If there exists an algebraic automorphism $\psi: \operatorname{Alg}\{G(-A), G(A)\} \rightarrow$ $\operatorname{Alg}\{G(-A), G(A)\}$ such that $\psi(B) T \xi=T B \xi$, for every $B \in \operatorname{Alg}\{G(-A), G(A)\}$ and every $\xi \in G(-A)+G(A)$, then there exists a scalar $\lambda$ and operators $R, S, D, E \in \mathscr{B}(H)$, with $D$ and $E$ invertible, such that $P-\lambda Q=R A, P^{-1}-$ $\frac{1}{\lambda} Q^{-1}=S A, A P=D A$, and $A Q=E A$.

Proof. Let $\psi$ be an automorphism as described in the statement of the lemma. If $A$ is invertible the desired result follows easily. Assume that $A$ is not invertible. Now

$$
\operatorname{Alg}\{G(-A), G(A)\}=\left\{\left(\begin{array}{cc}
A^{-1} W A & Z \\
A Z A & W
\end{array}\right): Z \in \mathscr{B}(H) \text { and } W \in \mathscr{A}(A)\right\},
$$

where, as before, $\mathscr{A}(A)=\{W \in \mathscr{B}(H): W A=A X$, for some $X \in \mathscr{B}(H)\}$. Let $W \in \mathscr{A}(A)$ with $W A=A X$, put $B=\left(\begin{array}{cc}X & 0 \\ 0 & W\end{array}\right)$ and let $\psi(B)=\left(\begin{array}{cc}X^{\prime} & Z^{\prime} \\ A Z^{\prime} A & W^{\prime}\end{array}\right)$. From the fact that $\psi(B) T \xi=T B \xi$, for every $\xi \in G(-A)+G(A)$, it follows that

$$
\left(X^{\prime}-Z^{\prime} A\right) P=P X, \quad\left(X^{\prime}+Z^{\prime} A\right) Q=Q X .
$$


Hence $A P X=A\left(X^{\prime}-Z^{\prime} A\right) P=\left(W^{\prime}-A Z^{\prime}\right) A P$, so

$$
X^{*}\left(P^{*} A\right)=P^{*} A\left(W^{\prime}-A Z^{\prime}\right)^{*} .
$$

It follows that $\mathscr{R}\left(P^{*} A\right)$ is invariant under every element of $\mathscr{A}(A)$, so $\mathscr{R}(A) \subseteq$ $\mathscr{R}\left(P^{*} A\right)$. Hence $A=P^{*} A X_{1}$, for some $X_{1} \in \mathscr{B}(H)$. Now the action of $T^{-1}$ on $G(-A)+G(A)$ is given by

$$
T^{-1}\left(\begin{array}{c}
x+y \\
A(y-x)
\end{array}\right)=\left(\begin{array}{c}
P^{-1} x+Q^{-1} y \\
A\left(Q^{-1} y-P^{-1} x\right)
\end{array}\right)
$$

and $\psi^{-1}(B) T^{-1} \xi=T^{-1} B \xi$, for every $B \in \operatorname{Alg}\{G(-A), G(A)\}$ and every $\xi \in$ $G(-A)+G(A)$. By what has just been proved, $A=P^{*-1} A X_{2}$, for some $X_{2} \in$ $\mathscr{B}(H)$. We have $A=A X_{1} X_{2}=A X_{2} X_{1}$ so $I=X_{1} X_{2}=X_{2} X_{1}$. Thus $A P=$ $D A$, for some invertible operator $D \in \mathscr{B}(H)$. Similarly $A Q=E A$, for some invertible operator $E \in \mathscr{B}(H)$. From (4) we obtain

$$
P X P^{-1}-Q X Q^{-1}=-2 Z^{\prime} A
$$

so

$$
\left(Q^{-1} P\right) X-X\left(Q^{-1} P\right)=-2 Q^{-1} Z^{\prime} A P=\left(-2 Q^{-1} Z^{\prime} D\right) A
$$

and, taking adjoints,

$$
X^{*}\left(Q^{-1} P\right)^{*}-\left(Q^{-1} P\right)^{*} X^{*}=A\left(-2 Q^{-1} Z^{\prime} D\right)^{*} .
$$

Thus, for every $W \in \mathscr{A}(A)$ there exists $C \in \mathscr{B}(H)$ such that $W\left(Q^{-1} P\right)^{*}-$ $\left(Q^{-1} P\right)^{*} W=A C$. By Theorem $1,\left(Q^{-1} P\right)^{*}=\lambda_{1}+A R_{1}$, for some scalar $\lambda_{1}$ and some $R_{1} \in \mathscr{B}(H)$. This gives $P-\lambda Q=R A$ with $\lambda=\bar{\lambda}_{1}, R=Q R_{1}^{*}$. Applying this argument to $T^{-1}$ gives that $P^{-1}-\gamma Q^{-1}=S A$, for some scalar $\gamma$ and some $S \in \mathscr{B}(H)$. We have $P=\lambda Q+R A$ and $P^{-1}=\gamma Q^{-1}+S A$ so

$$
I=(\lambda Q+R A)\left(\gamma Q^{-1}+S A\right)=\lambda \gamma+F A
$$

where $F=\lambda Q S+\gamma R E^{-1}+R A S$. Thus $I-\lambda \gamma=F A$ and since $A$ is not invertible, $\gamma=\frac{1}{\lambda}$. This completes the proof.

Remark. In the statement of the above lemma the definition of $T$ can be replaced by

$$
T\left(\begin{array}{c}
x+y \\
A(y-x)
\end{array}\right)=\left(\begin{array}{c}
P x+Q y \\
A(P x-Q y)
\end{array}\right) .
$$

The same conclusion holds, with a similar proof.

Theorem 2. Let $A$ be a positive injective operator acting on a separable Hilbert space $H . \quad$ Every algebraic automorphism $\varphi: \operatorname{Alg}\{G(-A), G(A)\} \rightarrow$ $\operatorname{Alg}\{G(-A), G(A)\}$ is spatially induced, that is, there exists an invertible operator $T_{0} \in \mathscr{B}(H \oplus H)$ such that $\varphi(B)=T_{0} B T_{0}^{-1}$, for every $B \in \operatorname{Alg}\{G(-A), G(A)\}$.

Proof. Let $\varphi$ be an algebraic automorphism as described in the statement of the theorem. By [5, Theorems 4.5, 4.8] (see also [4, Theorem 6, p. 85]) there exists a linear transformation $T: G(-A)+G(A) \rightarrow G(-A)+G(A)$, injective and continuous on each of $G(-A), G(A)$ such that $\varphi(B) T \xi=T B \xi$, for every $\xi \in$ $G(-A)+G(A)$ and every $B \in \operatorname{Alg}\{G(-A), G(A)\}$. Also, $T$ satisfies either (a) $T G(-A)=G(-A), T G(A)=G(A)$ or (b) $T G(-A)=G(A), T G(A)=G(-A)$. 
Suppose that (a) holds. Since $T$ is a continuous bijection on $G(-A)$ and on $G(A)$, there exist invertible operators $P, Q \in \mathscr{B}(H)$ such that

$$
T\left(\begin{array}{c}
x+y \\
A(y-x)
\end{array}\right)=\left(\begin{array}{c}
P x+Q y \\
A(Q y-P x)
\end{array}\right)
$$

for every $x, y \in H$. By the preceding lemma, there exists a scalar $\lambda$ and operators $R, S, D, E \in \mathscr{B}(H)$, with $D$ and $E$ invertible, such that $P-\lambda Q=$ $R A, P^{-1}-\frac{1}{\lambda} Q^{-1}=S A, A P=D A$, and $A Q=E A$. The operator $T_{0}$ defined by

$$
T_{0}=\frac{1}{2}\left(\begin{array}{cc}
P+\lambda Q & -R \\
-A R A & D+\lambda E
\end{array}\right)
$$

is invertible, with inverse

$$
T_{0}^{-1}=\frac{1}{2}\left(\begin{array}{cc}
P^{-1}+\frac{1}{\lambda} Q^{-1} & -S \\
-A S A & D^{-1}+\frac{1}{\lambda} E^{-1}
\end{array}\right)
$$

and, for every $x, y \in H$,

$$
T_{0}\left(\begin{array}{c}
x+y \\
A(y-x)
\end{array}\right)=T\left(\begin{array}{c}
x+\lambda y \\
A(\lambda y-x)
\end{array}\right) .
$$

It follows that, for every $B \in \operatorname{Alg}\{G(-A), G(A)\}$ and every $x, y \in H$

$$
T_{0} B\left(\begin{array}{c}
x+y \\
A(y-x)
\end{array}\right)=T B\left(\begin{array}{c}
x+\lambda y \\
A(\lambda y-x)
\end{array}\right) .
$$

Hence,

$$
\begin{aligned}
\varphi(B) T_{0}\left(\begin{array}{c}
x+y \\
A(y-x)
\end{array}\right) & =\varphi(B) T\left(\begin{array}{c}
x+\lambda y \\
A(\lambda y-x)
\end{array}\right) \\
& =T B\left(\begin{array}{c}
x+\lambda y \\
A(\lambda y-x)
\end{array}\right)=T_{0} B\left(\begin{array}{c}
x+y \\
A(y-x)
\end{array}\right) .
\end{aligned}
$$

Since $G(-A)+G(A)$ is dense in $H \oplus H$, it follows that $\varphi(B)=T_{0} B T_{0}^{-1}$, for every $B \in \operatorname{Alg}\{G(-A), G(A)\}$.

Finally, suppose that (b) holds. In this case there exist invertible operators $P, Q \in \mathscr{B}(H)$ such that

$$
T\left(\begin{array}{c}
x+y \\
A(y-x)
\end{array}\right)=\left(\begin{array}{c}
P x+Q y \\
A(P x-Q y)
\end{array}\right)
$$

for every $x, y \in H$. Once again $P-\lambda Q=R A, P^{-1}-\frac{1}{\lambda} Q^{-1}=S A, A P=D A$, and $A Q=E A$, for some scalar $\lambda$ and operators $R, S, D, E \in \mathscr{B}(H)$, with $D$ and $E$ invertible (see the remark following the lemma above). In a similar fashion it can be shown that $\varphi$ is spatially induced by the invertible operator

$$
T_{1}=\frac{1}{2}\left(\begin{array}{cc}
P+\lambda Q & -R \\
A R A & -(D+\lambda E)
\end{array}\right)
$$

with inverse

$$
T_{1}^{-1}=\frac{1}{2}\left(\begin{array}{cc}
P^{-1}+\frac{1}{\lambda} Q^{-1} & S \\
-A S A & -\left(D^{-1}+\frac{1}{\lambda} E^{-1}\right)
\end{array}\right) .
$$

This completes the proof.

The following corollary is added for the sake of completeness. It answers affirmatively, at least on separable space, a question raised in [7]. 
Corollary. Let $\left\{M_{1}, N_{1}\right\}$ and $\left\{M_{2}, N_{2}\right\}$ be pairs of subspaces of a separable Hilbert space $H$ with $M_{i}$ and $N_{i}$ in generalized generic position $(i=1,2)$. Every algebraic isomorphism $\varphi: \operatorname{Alg}\left\{M_{1}, N_{1}\right\} \rightarrow \operatorname{Alg}\left\{M_{2}, N_{2}\right\}$ is spatially induced, that is, for every such $\varphi$ there exists an invertible operator $T_{0} \in \mathscr{B}(H)$ such that $\varphi(B)=T_{0} B T_{0}^{-1}$, for every $B \in \operatorname{Alg}\left\{M_{1}, N_{1}\right\}$.

\section{REFERENCES}

1. K. R. Davidson, Nest algebras, Pitman Res. Notes in Math., vol. 191, Longman, England, 1988.

2. P. A. Fillmore and J. P. Williams, On operator ranges, Adv. in Math. 7 (1971), 254-281.

3. P. R. Halmos, Two subspaces, Trans. Amer. Math. Soc. 144 (1969), 381-389.

4. M. S. Lambrou, On some reflexive lattices and related algebras, Dissertation, University of London King's College, 1977.

5. __ Automatic continuity and implementation of homomorphisms, preprint.

6. E. Nordgren, M. Radjabalipour, H. Radjavi, and P. Rosenthal, On invariant operator ranges, Trans. Amer. Math. Soc. 251 (1979), 389-398.

7. M. Papadakis, On isomorphisms between certain non-CSL algebras, Proc. Amer. Math. Soc. 119 (1993), 1157-1164.

Department of Mathematics, University of Crete, Iraklion, Crete, Greece

E-mail address: lambrou@talos.cc.uch.gr

Department of Mathematics, University of Western Australia, Nedlands, Western Australia 6009, Australia

E-mail address: longstaf @maths . uwa . edu . au 